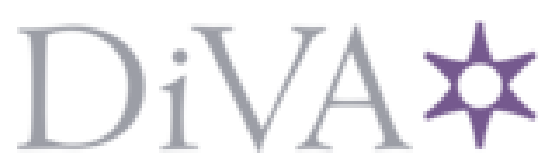

http://www.diva-portal.org

This is the published version of a paper published in Nordic Pulp \& Paper Research Journal.

Citation for the original published paper (version of record):

Hyll, C., Vomhoff, H., Mattsson, L. (2012)

A method for measurement of the directional emittance of paper in the infrared wavelength range.

Nordic Pulp \& Paper Research Journal, 27(5): 958-967

http://dx.doi.org/10.3183/NPPRJ-2012-27-05-p958-967

Access to the published version may require subscription.

N.B. When citing this work, cite the original published paper.

Permanent link to this version:

http://urn.kb.se/resolve?urn=urn:nbn:se:kth:diva-105415 


\section{A method for measurement of the directional emittance of paper in the infrared wavelength range}

\author{
Caroline Hyll, Hannes Vomhoff, and Lars Mattsson
}

KEYWORDS: Thermography, Method development, Paper emittance, Directional emittance, Hemispherical emittance, Emissivity, Goniometer, FTIR

SUMMARY: A method for measuring the directional emittance of paper and board samples was developed. The available literature showed that the influence of temperature and observation angle on the emittance of dry and moist paper had not been investigated in detail. Methods adapted for such investigations were not available.

In the developed method, the emittance of a sample is determined by comparing its infrared radiation with the radiation emitted from a reference surface with known emittance. In order to investigate the influence of the wavelength range, two cameras, operating in the midwavelength and long-wavelength infrared range, respectively, were used. The method allows for the adjustment of the directional emission angle in a range from $0^{\circ}$ down to $80^{\circ}$, and variation of the sample temperature between $30^{\circ} \mathrm{C}$ and $100^{\circ} \mathrm{C}$.

A study was performed to evaluate the method. Here, the directional emittance of handsheets made from thermo-mechanical pulp was measured at different wavelength ranges, sample temperatures and emission angles. The obtained emittance values and trends were in agreement with previous experimental work and theoretical predictions. The emittance of the samples was also measured using Fourier-Transform Infrared spectroscopy. Given the methodological differences between the two measurement approaches, the results were in good agreement.

ADDRESSES OF THE AUTHORS: Hyll, Caroline (caroline.hyll@innventia.com), Innventia AB, Drottning Kristinas väg 61, SE-114 28, Stockholm, and Industrial Metrology and Optics, Production Engineering, KTH Royal Institute of Technology, SE-100 44 Stockholm. Vomhoff, Hannes (hannes.vomhoff@innventia.com), Innventia AB, Drottning Kristinas väg 61, SE-114 28, Stockholm. Mattsson, Lars (larsm@iip.se), Industrial Metrology and Optics, Production Engineering, KTH Royal Institute of Technology, SE-100 44 Stockholm.

\section{Corresponding author: Caroline Hyll}

Thermography is a technique for non-destructive inspection, evaluation and testing of materials and processes. Within the pulp and paper industry, it has been used on many occasions, for example for analysis of the production process (Sheahen 1978; Vickery et al. 1978; Charles 1999), or research related to different product properties (Kiiskinen and Pakarinen 1998; Yamauchi and Murakami 1992; Hojjatie et al. 2001; Dumbleton et al. 1973).

In thermography, infrared detectors are used to measure the radiation emitted by objects due to their temperature (e.g. DeWitt and Nutter 1988; Vollmer and Möllmann 2010). The radiation can be converted to a temperature measurement using the Planck's law. Here, the emitted radiance as a function of wavelength and temperature are related for an ideal radiator, i.e. a blackbody. Most real objects are only emitting a fraction of the radiation that a blackbody would emit under identical conditions. This fraction, the emissivity, is a parameter unique to each material. When measured on samples where the limited thickness can influence the results, the emissivity is called emittance (McCluney 1994), in analogy with resistivity of a material and resistance of a limited piece of it. The emittance of a material may vary with emission angle, wavelength, and the temperature, structure and composition of the material. A value of the emittance must be provided to infrared cameras if temperature output values are to be obtained.

Using the correct value for the emittance is crucial, as an absolute error of $15 \%$ in the emittance could introduce an error of $3 \%$ to the measured object temperature in Kelvin (Minkina and Dudzik 2009). For example, using an emittance of 0.82 when the true emittance is 0.71 would cause an error of $10 \mathrm{~K}$, if the measured object temperature is $343 \mathrm{~K}\left(70^{\circ} \mathrm{C}\right)$.

Despite its importance for the quantitative measurement result, the emittance values were only stated in a few publications where thermography was used for pulp and paper industry applications (Banerjee et al. 2008; Charles 1999; Fike 2004; Banerjee 2008). This can be explained partly by the fact that the majority of the studies were qualitative investigations rather than quantitative measurements.

Methods for measuring emittance comprise standardized (ASTM 2008; ASTM 2004) and nonstandardized methods and equipment (e.g. Puram et al. 1994; Madding 1999; Beecroft et al. 2005). Most experimental work on the determination of the emittance of paper used non-standardized methods (Trepanier 1984; Öhman 1999; Ojala 1993). Non-standardized methods include reflectivity methods, reference emitter methods and reference temperature methods. The methods may either measure the directional emittance, which is measured at a specific emission angle (usually normal to the surface), or the hemispherical emittance, which is integrated over all possible directions in the hemisphere above the object. Reflectivity methods determine emittance indirectly by measuring the reflectance and transmittance of the object and relating those properties to the emittance. Most reflectivity method measurements are performed with Fourier Transform Infrared spectroscopy (FTIR), which usually measures the hemispherical emittance. For additional information on FTIR spectroscopy see e.g. Griffiths \& De Haseth (1986), or Smith (2011). Reference emitter methods determine emittance by comparing the radiation of the object to that 
of a reference under identical conditions. Here, the emittance of the reference needs to be known (Öhman 2001). Reference temperature methods determine the emittance of an object under the requirement that the temperature of the object is well known. Reference emitter and reference temperature methods require the sample to be opaque (have a transmittance of zero) but can be carried out with normal infrared cameras provided that the environment is well-controlled. Furthermore, the sample has to be heated to temperatures above ambient temperature (Öhman 2001).

Since paper is a dielectric (insulator) the behaviour of paper emissivity is expected to conform to the general behaviour of dielectrics. Here, the emissivity is expected to decrease with increased temperature (Gaussorgues 1994; Öhman 2001), increase with increased wavelength (Gaussorgues 1994; Öhman 1999), and behave roughly constant down to approximately $50^{\circ}$ observation angle from the normal angle $\left(0^{\circ}\right)$, where it starts to decrease (Maldague 2001).

The most detailed investigations on the emittance of paper were performed by Trepanier (1984) and Öhman (1999). In addition, Ojala et al. (1992) and Lescanne (1992) measured the absorptance of paper, which according to Kirchoff's law equals emittance. Furthermore, in the widely referenced work of Bramson (1968), where several Russian sources of thermographyrelated material are collected, graphs are included that show the emittance of paper as a function of observation angle and temperature. However, it is not clear if the graph showing paper emittance and angle are based on theoretical or experimental work. Furthermore, emittance values are shown at temperatures that are well above the self-ignition temperature of paper (around $505 \mathrm{~K}$, or $230^{\circ} \mathrm{C}$ ). Bramson also collects some Russian sources containing values for the normal emittance of paper, but does not give the spectral range of the measurements. Due to these unclarities, the results presented in Bramson (1968) will not be used as references in the present study.

Trepanier (1984) used a reference temperature method to carry out his pioneering work on the normal emittance of dry and moist paper for different paper grades and grammages. Trepanier also studied the influence of onesided and two sided drying and calendaring on the emittance of paper. The measurements were performed in the 3.0-5.6 $\mu \mathrm{m}$ wavelength range (AGA 1979; Trepanier 1984).

Öhman (1999) used a reference emitter method to measure the normal emittance of a wide range of materials, including paper products, in two different wavelength ranges; 2.0-5.4 $\mu \mathrm{m}$ and 8.0-12 $\mu \mathrm{m}$ (AGEMA 1994; Öhman 1999).

Ojala et al. (1992) used FTIR spectroscopy to measure the hemispherical transmittance and reflectance of coated paper at wavelengths $1-20 \mu \mathrm{m}$. The values of the transmittance and reflectance were used to calculate the emittance of their samples. In 1993, Ojala used the same setup to study the impact of grammage and moisture content on the transmittance, reflectance and absorptance of their samples.

Lescanne (1992) used a reflectivity method to measure the hemispherical transmittance and reflectance of softwood paper at wavelengths 2.5-15.0 $\mu \mathrm{m}$. The emittance was then calculated from the reflectance and transmittance values in the same way as Ojala (1992).

No literature was found where measurement results of the directional sensitivity of the emissivity of paper were reported. For other natural surfaces some investigations have been carried out for the purpose of thermal remote sensing. Labed and Stoll (1991) measured the angular dependence of the emissivity of soils and sand at wavelengths $3.0-13.5 \mu \mathrm{m}$. They found that the sensitivity to observation angle was higher at shorter infrared wavelengths than at longer infrared wavelengths. Additionally, it was found that the emissivity was approximately constant down to an angle of $60^{\circ}$ from the normal, where it started to decrease. For longer infrared wavelengths some samples showed an increase in emissivity around $50^{\circ}$ from the normal, before the emissivity started to drop.

Based on the previously published work, it can be concluded that the influence of temperature and observation angle on the emittance of dry and moist paper has not been investigated in detail. Furthermore, detailed investigations on the influence of the raw material composition (type of pulp, filler) and pulp treatment have not been performed. In several published studies, the hemispherical emittance of paper was studied. However, thermographic cameras only detect radiation emitted in the direction of the camera sensor. During practical thermographic applications the observation angle between the camera and the target may vary, for example when imaging a pope roll or a drying cylinder Values for the directional emittance would therefore be more relevant.

The objective of the present study was therefore to develop a new method for measuring the directional emittance of paper. The method should allow for investigation of how observation angle, wavelength range, temperature, raw material composition and moisture content influence the emittance. Furthermore, the measurement should be performed using a thermographic camera in order to get relevant emittance values.

\section{Theory and definitions}

All objects with temperature above the absolute zero emit radiation. For a wide range of object temperatures, most of the radiation is emitted in the infrared (Maldague 2001). The infrared part of the electromagnetic spectrum is loosely defined as covering wavelengths between 0.8 and $1000 \mu \mathrm{m}$. Commonly, two wavelength ranges, known as the Mid-Wavelength Infrared ("MWIR", approximately 3-6 $\mu \mathrm{m})$ and the Long-Wavelength Infrared ("LWIR", approximately $8-15 \mu \mathrm{m}$ ) are used in thermography. Detection outside of these ranges is limited due to sensor material properties and atmospheric absorption.

The relationship between object temperature and wavelength of the emitted radiation can be explained by Planck's law. For any wavelength $\lambda$ in the electromagnetic spectrum, Planck's law relates the emitted spectral radiance $L_{b b \lambda}$ of a true blackbody to the 


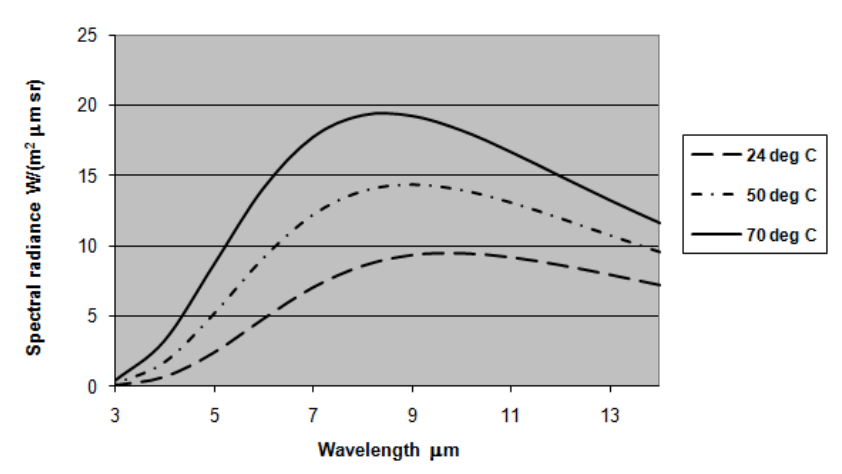

Fig 1. Spectral radiance as a function of wavelength at room temperature and temperatures $50^{\circ} \mathrm{C}$ and $70^{\circ} \mathrm{C}$.

temperature $T$ of the emitting object. For a small wavelength interval $\mathrm{d} \lambda$, it is expressed as:

$$
L_{b b \lambda} d \lambda=\frac{2 h c^{2}}{\lambda^{5}\left(e^{h c / \lambda k T}-1\right)} d \lambda
$$

where $h$ is Planck's constant, $c$ is the speed of light in vacuum, $\lambda$ is the wavelength at which the light is emitted, and $k$ is Boltzmann's constant.

Using $E q[1]$ the spectral radiance for a perfect blackbody has been calculated and the spectral radiance is shown in Fig 1 for three temperatures; room temperature at $24^{\circ} \mathrm{C}$, a temperature of $50^{\circ} \mathrm{C}$ and a temperature of $70^{\circ} \mathrm{C}$.

Fig 1 indicates that when measuring an object with a temperature of $70^{\circ} \mathrm{C}$ in a room where the ambient temperature is $24^{\circ} \mathrm{C}$, the background radiation (or "noise") will be nearly half that of the object signal. It is thus important to measure and compensate for the background (or "ambient") radiation.

Distinguishing the background radiation from the radiation emitted by the object can be done with knowledge of the object's reflectance, transmittance and absorptance. For a general material with a smooth surface and a thick bulk volume the reflectivity $\rho$ is the fraction of the reflected radiation in relation to the incident flux. Correspondingly, the transmissivity $\tau$ and absorptivity $\alpha$ are the fractions of the transmitted and absorbed radiation, respectively, in relation to the incident flux. Values for the reflectance, transmittance, and absorptance can be measured experimentally. They are materialdependent and in the range of 0 to 1 . In addition, their sum has to be equal to 1 :

$$
a+\tau+\rho=1
$$

Under thermodynamic equilibrium, all absorbed radiation is re-emitted and the absorptivity is equal to the emissivity (Kirchoff's law):

$$
\alpha=\varepsilon
$$

Emissivity and absorptivity are also equal under nonequilibrium conditions provided that their values are given for the same wavelength and direction (Dewitt and Nutter 1988). This equality is often used to derive emittance values from measured reflectance and transmittance measurements. In the present work it is therefore assumed that absorptance is equal to emittance

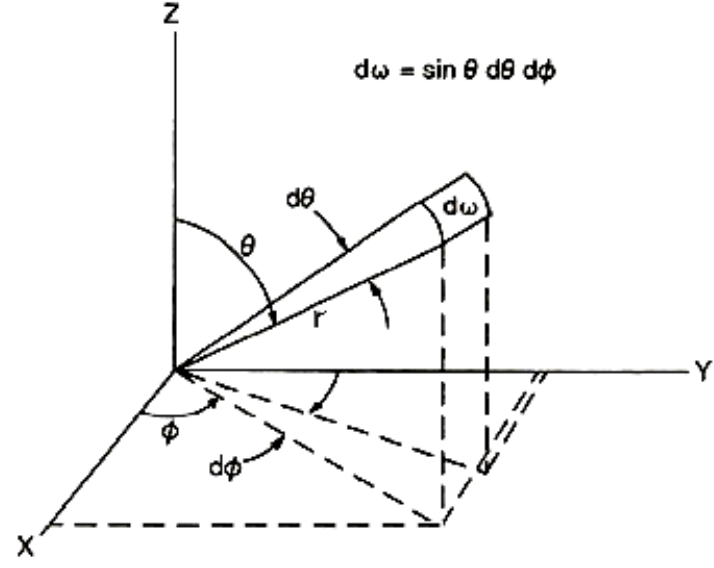

Fig 2. The directional emittance is derived from radiance measurements within the solid angle $d \omega$ at the observation angle $\theta$ relative to the surface normal (z-axis) and the azimuthal angle $\Phi$ relative the XZ-plane.

when using literature sources where absorptance is given but not emittance.

Finally, thermography only detects radiation emitted in the direction of the camera sensor, within the wavelength range where the sensor detects radiation, and within a solid angle $d \omega$ determined by the entrance pupil of the camera lens. A material might emit radiation differently in different directions, e.g. the radiance can be dependent on the polar observation angle $\theta$ and azimuthal angle $\Phi$, see Fig 2. Consequently, the emittance may vary with emission angle. As mentioned in the previous section it can be measured as either directional or hemispherical emittance, dependent on measurement method. Hemispherical emittance is mainly used for radiative heat transfer calculations.

\section{Experimental}

A "Directional Emittance Measurement" (DEM) method was developed in the present work. It is based on the reference emitter methodology, i.e. the radiation from a sample is compared to that from a reference with known emittance under otherwise identical conditions.

In FLIR System infrared cameras the emitted radiance from an object generates a camera output signal voltage $U$, in volts, that is proportional to the power input, i.e. the camera is "power linear" (Öhman 2001). The signal voltage can be directly obtained as output data from the camera, given in "S-units". Alternatively, the camera can automatically convert the output signal to a temperature value through a calibration procedure, if parameters such as the emittance are given. When the emittance is to be determined, signal voltage is the necessary output data to be obtained from the camera.

In the developed method, paper samples and emittance reference are placed inside an oven whose temperature can be controlled. The infrared camera is mounted onto a goniometer, e.g. a holder that can be pivoted around the oven in order to change the observation angle. When the desired oven temperature is reached, the lid of the oven is opened and the samples are imaged with the infrared camera. 


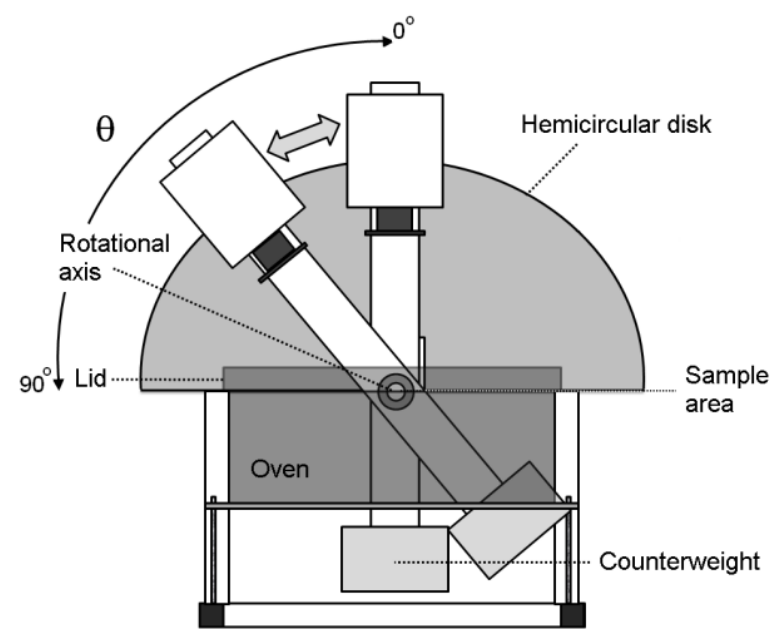

Fig 3. Illustration of the Directional Emittance Measurement (DEM) setup showing the goniometer and the oven.

Ambient radiation emitted from the surroundings is measured by imaging a high-reflectance surface at the same position as the samples. The emittance of the sample material can then be determined based on the measured directional radiance of the sample, highreflectance surface, and reference, according to:

$$
\varepsilon_{\text {sample }}=\varepsilon_{\text {ref }} \frac{U_{\text {sample }}-U_{a m b}}{U_{\text {ref }}-U_{a m b}}
$$

where $\varepsilon_{r e f}$ is the emittance of the reference, $U_{\text {sample }}$ is the signal voltage from the camera due to the emitted directional radiance of the sample, $U_{a m b}$ is the signal voltage from the camera due to the emitted radiance of ambient surroundings, reflected into the camera by a high-reflectance surface, and $U_{r e f}$ is the signal voltage from the camera due to the emitted radiance of a wellestablished emittance reference. Here, it is assumed that the scattering properties of the paper samples, the highreflectance surface and the reference are identical.

$E q[4]$ is valid for opaque objects. Its derivation can be found in Öhman (2001).

\section{Goniometer for radiance measurements}

The DEM method comprises an oven, a goniometer for the positioning of the camera at the right observation angle $\theta$, an infrared camera, an inclinometer, a highreflectance rough gold surface with lambertian scatter distribution, and an emittance reference; see Fig 3 and Fig 4.

Using the goniometer, the camera can be positioned to an arbitrary angular position $\theta$ on a hemicircular metal plate. The position of the camera is locked using a brake. The system is stabilized by a counter-weight allowing camera weights up to $10 \mathrm{~kg}$. The oven is connected by an arrangement to the goniometer. The sample location in the oven is positioned at the same height as the rotational axis of the inclination positioned. The centre of the sample area is on the same height as the rotational axis of the inclination positioner. This minimizes length projections of the sample area as the angle is varied. The samples are heated using a specially-designed oven developed by AGEMA Infrared Systems (Öhman 1996;

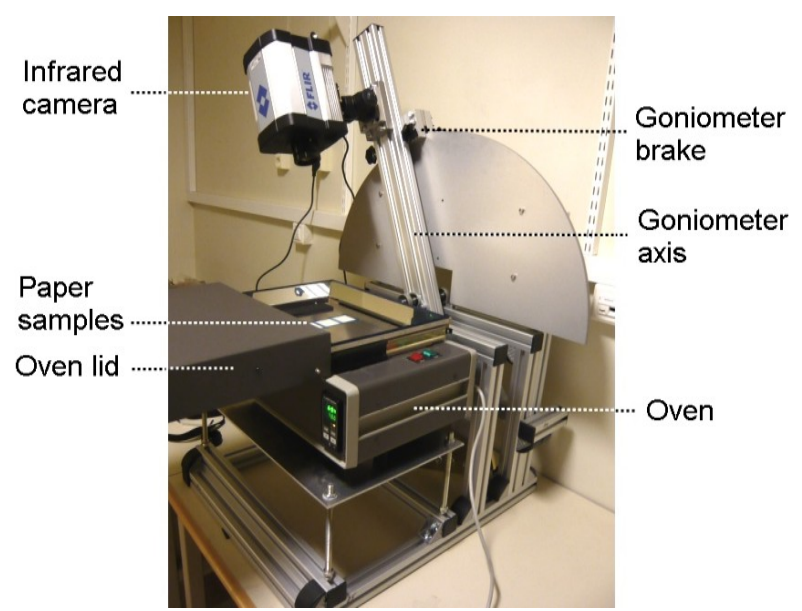

Fig 4. Image of the directional emittance measurement setup, including the goniometer and oven.

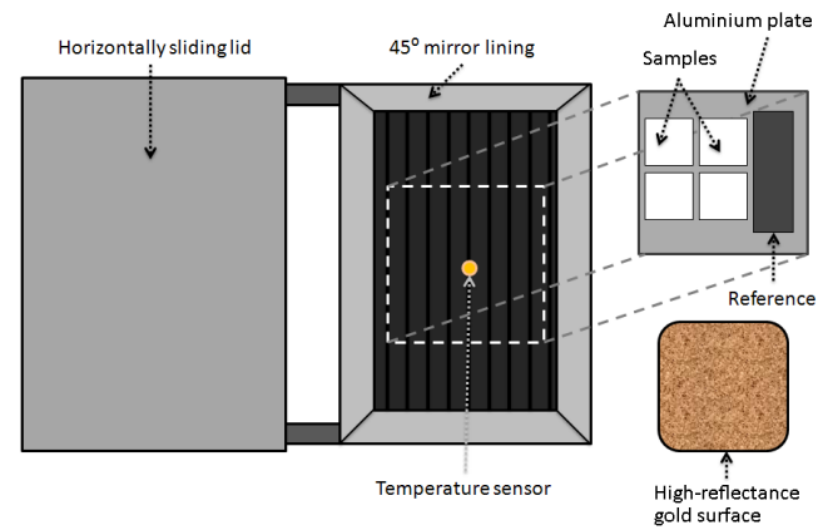

Fig 5. Illustration of oven with the horizontally sliding lid, the $45^{\circ}$ inclined mirror linings, temperature sensor (located below the aluminium plate), aluminium plate (samples and Nextel emittance reference), and the high-reflectance gold surface.

Öhman 2001). An illustration of the oven is shown in Fig 5. The oven was designed to heat the samples homogeneously prior to the emittance measurements. Furthermore, the influence of radiation emitted by the interior of the oven onto the sample area is minimized. Such radiation would introduce a significant error to the measurement results. Radiation from the oven walls onto the sample during a measurement is minimized by the interior mirror lining and the horizontally sliding lid. Having a horizontal lid instead of a vertical lid is also expected to reduce temperature changes due to air flows during the opening of the lid.

Stable and even heating is achieved by a combination of a heating element and a fan. The temperature is set in the control unit. A temperature sensor, located just below the aluminium plate, measures the temperature of the sample. This temperature reading is used in the control loop of the control unit. According to Öhman (1996), the oven temperature has an accuracy of $\pm\left(0.3^{\circ} \mathrm{C}+0.004^{*} \mathrm{~T}\right)$, where $\mathrm{T}$ is the set temperature in ${ }^{\circ} \mathrm{C}$.

The observation angle $\theta$ is measured by the Digital Angle Gauge from Wixley (0 to $180^{\circ}$ range, $0.1^{\circ}$ resolution, $\pm 0.1^{\circ}$ accuracy). Ambient radiation is measured using a high-reflectance lambertian gold surface; Infragold 5' x 5' from Labsphere (reflectance 
0.94-0.96 at $\lambda=2-20 \mu \mathrm{m})$. Emittance reference comprises a plate of rugged aluminium painted with five layers of Nextel Velvet 811-12 from Mankiewicz Corp. Nextel Velvet 811-12 has a high emittance which is well-studied for several wavelengths, angles and temperatures (Koirala 2004; Kwor and Matteï 2001). In this study a Nextel emittance value of 0.92 was used for the MWIR measurements, and a value of 0.94 was used for the LWIR measurements (see following sections).

Two cameras were used for evaluation. The FLIR SC6000 camera (www.flir.com) has a cooled IR-detector and operates in the MWIR range (3.0-5.0 $\mu \mathrm{m})$. According to the company's data sheet, the accuracy of the absolute temperature reading is $\pm 2.0^{\circ} \mathrm{C}$, or $\pm 2.0 \%$ for other kinds of output (such as signal voltage). SC6000 was used with a $25 \mathrm{~mm}$ lens. The FLIR SC640 (www.flir.com) has an uncooled detector and operates in the LWIR range (7.5$13.0 \mu \mathrm{m})$. It is specified to have the same measurement accuracy as the SC6000. The SC640 was used with a 38 mm lens.

The paper samples for the initial study comprised standard handsheets (ISO 5269-1:2005) made from Thermo-mechanical pulp (TMP, $75.0 \mathrm{ml} \mathrm{CSF}$ ), that is used for producing improved newsprint. The $16 \times 16 \mathrm{~cm}^{2}$ sized sheets had a grammage $(w)$ of $60 \mathrm{~g} / \mathrm{m}^{2}$. The sheets were assumed to have an isotropic structure. If a surface is isotropic the emittance can be assumed to be constant with respect to azimuthal (in-plane) observation angle $\Phi$ (Dewitt and Nutter 1988). However the emittance may still vary with inclination angle $(\theta)$. If measurements were to be made on industrially manufactured sheets the assumption of isotropy might not be valid due to a more pronounced fibre orientation in the machine direction.

\section{Equipment for FTIR measurements}

In order to compare the emittance results obtained with the DEM method with results obtained with a different method, FTIR spectroscopic measurements were performed on the same samples. The measurements were performed with a single-beam spectrophotometer (Bruker Tensor 27). The equipment measures at room temperature and generates spectral-hemispherical (integrated over all angles) reflectance and transmittance spectra between wavelengths $2.5 \mu \mathrm{m}$ and $22.2 \mu \mathrm{m}$. The golden reflectance standard used to obtain calibration spectra had a reflectance of $0.98 \pm 0.02$.

\section{Experimental procedure of DEM measurements}

The measurements were executed in a windowless room. The light source in the room was selected to emit very little at infrared wavelengths, and was directed away from the measurement area. Stronger sources of infrared radiation in the room, such as hot water pipes, were shielded off using aluminium sheets. During the course of the measurements the air temperature was assumed to equal the ambient temperature (the temperature representative of the surroundings in the room). The average measured air temperature was $24^{\circ} \mathrm{C}$. During all measurements the samples were heated long enough to become bone dry, implying $98-99 \%$ solids content.

Four $5 \mathrm{~cm}$ x $5 \mathrm{~cm}$ samples were cut out from each TMP handsheet. The samples were taped onto a $5 \mathrm{~mm}$ thick aluminium plate using temperature resistant masking tape in an arrangement as shown in Fig 5. Special care was taken to eliminate air pockets between paper samples and plate which would otherwise cause local variations in conductive heat transfer. The Nextel-painted reference plate was placed next to the paper samples. The sensor of the camera was corrected for local non-uniformity according to a FLIR standard procedure and set to a frame rate of $15 \mathrm{~Hz}$.

When evaluating the temperature dependency of the emittance, the reference plate and the samples were placed in the oven and heated to temperatures between $30^{\circ} \mathrm{C}$ and $100^{\circ} \mathrm{C}$, in $10^{\circ} \mathrm{C}$ intervals. The camera was mounted at normal observation angle $\left(\theta=0^{\circ}\right)$ with 220 or $500 \mathrm{~mm}$ distance, dependent on the type of camera, between objective and sample surface. The oven was heated until reaching equilibrium at the desired temperature, and then heated an additional $5 \mathrm{~min}$ to assure an even temperature distribution. The fan was then turned off and the oven was allowed to rest for $20 \mathrm{~s}$ to stabilize the internal air flow. Video recording was started and the lid of the oven was opened, exposing the camera sensor to the samples. Approx. 0.3 to $0.5 \mathrm{~s}$ elapsed between lid opening and imaging of the samples. After the imaging of the samples the infrared highreflecting surface was placed in the field-of-view, imaged and subsequently removed. This procedure ensured that the reflecting surface had ambient temperature, giving an accurate measurement of the radiation from the surroundings. Placing, imaging and removing the reflecting surface took approximately $10 \mathrm{~s}$. The lid was then closed, the fan turned on, the temperature setting raised and the procedure described above repeated at the next measurement temperature.

For measurements of the angular dependent emittance, samples were put into the oven and heated to $70^{\circ} \mathrm{C}$. The camera was mounted at normal observation angle and subsequently inclined in $10^{\circ}$ intervals down to $80^{\circ}$. Video recording for each angle was performed with the same procedure as described above.

To evaluate the spectral variation in directional emittance, the angular- and temperature-dependent measurement were performed using the two different cameras; the FLIR SC6000 MWIR (sensitive between 3.0 and $5.0 \mu \mathrm{m}$ ) and the FLIR SC640 (sensitive between 7.5 and $13.0 \mu \mathrm{m})$.

To assess the repeatability of the method, each measurement investigating the angular, thermal and spectral variation was repeated five times.

To investigate the influence of a possible transparency and bulk effect of the samples, a measurement series was performed where one, three, and five TMP samples were put on top of each other. Two stacks of each were taped to the aluminium plate. The sample stacks were then heated to $70^{\circ} \mathrm{C}$ and imaged at normal observation angle. The measurements were repeated five times using the same samples.

Emittance data for the reference plate was not available for every angle. The emittance of the reference was thus determined through reference temperature measurements. The Nextel-painted reference plate was put in the oven and heated during 2 hours at $70^{\circ} \mathrm{C}$. Imaging was then 
performed with the same procedure as the angular dependent measurements.

\section{Data analysis of DEM measurements}

Camera recordings were evaluated using the ThermaCam Researcher Professional 2.9 software from FLIR Systems. Here, radiation power values were displayed as signal output voltage ("S-units"). From each sequence of images (corresponding to one measurement) only one frame (snapshot) was used for evaluation. This frame was chosen as the fourth frame after full oven lid opening (e.g. when the full scene is viewed by the camera), around 0.3 to $0.5 \mathrm{~s}$ after opening of the lid. This allows for some air stabilization after the opening while not giving the samples too much time to cool down.

One needs to consider cooling effects during analysis of the images. Paper loses heat quickly when the lid is removed. When the sample has been heated to a temperature of $70^{\circ} \mathrm{C}$ and the atmospheric temperature is $24^{\circ} \mathrm{C}$ the paper samples will cool 3.0 to $4.5^{\circ} \mathrm{C}$ per second after the lid has been opened. Typical times from lid opening to imaging of the samples are 0.3 to 0.5 seconds. Between lid opening and imaging, samples further away from the lid (e.g. the bottom row of samples in Fig 6) will have slightly more time to cool than samples closer to the lid. The Nextel-painted aluminium reference plate retains heat better and cools approx. $1.3^{\circ} \mathrm{C}$ per second. Since the reference and paper samples are imaged at the same time the reference plate will have a different temperature than the paper samples. The estimated error due to this effect was included in the error analysis.

The emittance of the reference was determined by the built-in reference temperature emittance determination feature of ThermaCam Researcher. The temperature of the reference was assumed to be $69.8 \pm 0.2^{\circ} \mathrm{C}$ in the first frame fully imaging the reference. The results were used to compensate for the spectral-, thermal- and angular dependence of the Nextel emittance reference. The normal emittance of the reference plate was found to be 0.92 in the MWIR and 0.94 in the LWIR. The measured directional behavior of the reference plate can be found in Hyll et al. (2011).

The emittance of the paper samples was determined through the following analysis. The average recorded signal value was taken over an approx. $2 \mathrm{~cm} \times 2 \mathrm{~cm}$ area in the centre of each paper sample, the emittance reference, and the high-reflectance gold surface (Fig 6).

The average emittance of each individual sample was calculated from Eq [4], using Matlab routines. The average emittance value of the four samples was taken as the result of the measurement. Each measurement was repeated five times and the mean value of the five measurements was determined and taken as the final result. The standard deviation of the fives measurement values was also determined.

A theoretical measurement error was estimated through mean error propagation of $E q$ [4]. Estimations of both random and systematic error were included in the estimation. The result of the error propagation indicated the LWIR measurements exhibit a greater theoretical

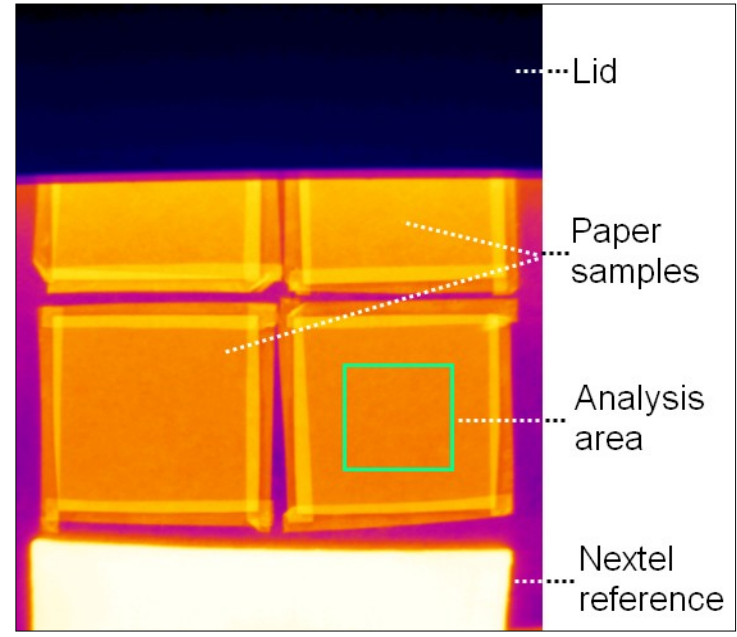

Fig 6. Example of an infrared image of four paper samples and Nextel reference plate during opening of the lid. The analysis area for the determination of the mean value is shown. Due to its higher emittance, the Nextel reference appears relatively brighter in the image compared to the paper samples. The image was taken with the FLIR SC6000 MWIR camera.

error than the MWIR measurements. In addition, it was found that - as expected - the largest theoretical errors occur for temperatures around ambient temperature (below $50^{\circ} \mathrm{C}$ ), as the numerator of $E q$ [4] approaches zero. The contribution to the error from the numerator should be random. The theoretical error should therefore be compared to the experimental standard deviation between measurement sets. A low experimental standard deviation even at temperatures below $50^{\circ} \mathrm{C}$ would indicate that the theoretical error is over-estimated. However, systematic errors could still be present. The estimated error in the emittance for temperatures above $50^{\circ} \mathrm{C}$ should give an estimation of the systematic error, since the effect of the numerator should be low at those temperatures.

The total error was taken to be the sum of the standard deviation of the five measurement sets (estimation of reliability) and the mean error given by the theoretical error propagation (estimation of accuracy) for temperatures above $50^{\circ} \mathrm{C}$.

\section{Experimental procedure and analysis of FTIR measurements}

Reflecting gold surfaceectance and transmittance measurements were performed separately. Each measurement was performed on two different samples made from the same pulp. The effective measurement area was $1 \mathrm{~cm}^{2}$. The output spectra from the FTIR equipment were the average of 100 scans on the same measurement

area.

The emittance spectrum was calculated from the reflectance- and transmittance spectra, under the assumption that the sum of reflectance, transmittance and emittance are equal to one at any given wavelength ( $E q$ [2]-[3]). The resulting emittance spectrum was then linearly averaged over the MWIR and LWIR wavelengths ranges, respectively, to obtain results comparable to those obtained with the DEM method. 


\section{Results and discussion}

The normal emittance of the stacks comprising one, three, and five TMP samples is presented in Table 1. First of all, we note that the normal emittance of TMP is higher at in the LWIR wavelength range than in the MWIR. This is in agreement with the results of Öhman (1999), Ojala (1992) and Lescanne (1992). Furthermore, the difference in emittance for different numbers of samples in the stacks was within the standard deviation of the measurements. It could therefore be concluded that one sample of TMP had sufficiently low transmittance in both the MWIR and the LWIR to be considered opaque at the measured wavelength range. In the following, measurements were therefore performed with only one sample.

The emittance of the TMP samples was also measured with hemispherical FTIR spectroscopy. FTIR spectroscopy measured over a range of discrete wavelengths and thus allowed the spectral behaviour of the emittance to be investigated in greater depth. The measured reflectance-, transmittance- and emittance spectra of TMP are shown in Fig 7.

The spectrally averaged results from the FTIR measurements are presented in Table 2.

The FTIR measurements confirmed the above-stated speculation of high reflectance and low transmittance of the TMP samples. When comparing the directional emittance (Table 1) and the hemispherical emittance (Table 2) there is a $6 \%$ difference in the MWIR wavelength range and a $2 \%$ difference in the LWIR. The similarity in results from the two methods is good, given the methodological differences. However the higher discrepancy in the MWIR is surprising. A possible factor that could create a difference between the two wavelength ranges is the roughness of the surface. The TMP samples have a notable surface roughness, in particular in a wavelength range related to the width of
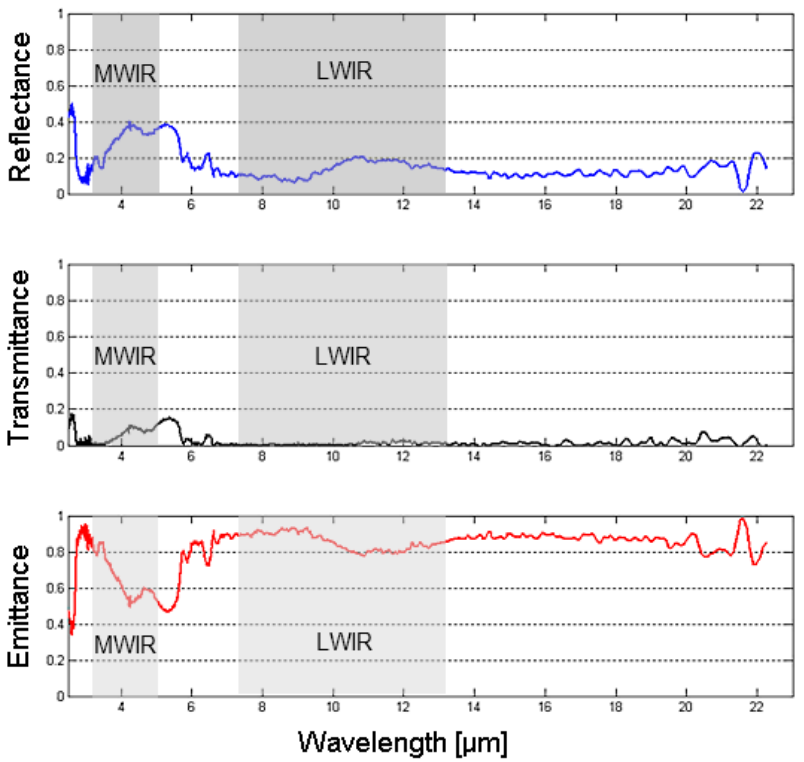

Fig 7. FTIR reflectance, transmittance and emittance spectra of TMP for improved newsprint. The MWIR and LWIR wavelength ranges are indicated.
Table 1. Normal emittance of TMP measured with the DEM method at temperature $70^{\circ} \mathrm{C}$. The results are averaged over five measurement sets. As error range, \pm the total error (standard deviation of the average + estimated accuracy) is given.

\begin{tabular}{lll}
\hline & MWIR & LWIR \\
\hline Stack of 1 sample & $0.61 \pm 0.03$ & $0.87 \pm 0.04$ \\
Stack of 3 samples & $0.61 \pm 0.03$ & $0.88 \pm 0.04$ \\
Stack of 5 samples & $0.61 \pm 0.03$ & $0.87 \pm 0.04$ \\
\hline
\end{tabular}

Table 2. Hemispherical reflectance, transmittance and emittance of TMP samples, measured with FTIR and averaged over wavelengths 3.0-5.0 $\mu \mathrm{m}$ (MWIR) and 7.5-13.0 $\mu \mathrm{m}$ (LWIR).

\begin{tabular}{lll}
\hline & MWIR & LWIR \\
\hline Reflectance $\rho$ & $0.28 \pm 0.02$ & $0.14 \pm 0.02$ \\
Transmittance $\mathbf{T}$ & $0.05 \pm 0.02$ & $0.01 \pm 0.02$ \\
Emittance $\boldsymbol{\varepsilon}$ & $0.67 \pm 0.03$ & $0.85 \pm 0.03$ \\
\hline
\end{tabular}

the fibres, approximately 20 to $55 \mu \mathrm{m}$. At shorter wavelengths the surface appears rougher from the perspective of the radiation than at longer wavelengths. The influence of the surface roughness should thus be less pronounced in the LWIR.

It is desirable to know how the results obtained with the DEM method relate to paper emittance values measured with similar or other methods. Directional and hemispherical emittance values of the literature and our study is shown in Fig 8 and Fig 9. First, results for the MWIR wavelength range are presented. A general observation is that the spread in measured emittance for the published works appear to be large. However when comparing the different studies, one needs to consider differences in paper type, raw material, grammage, and measurement methodology. From Fig 8 we note that Trepanier's result deviates from the remaining results by exhibiting a significantly higher emittance. A possible

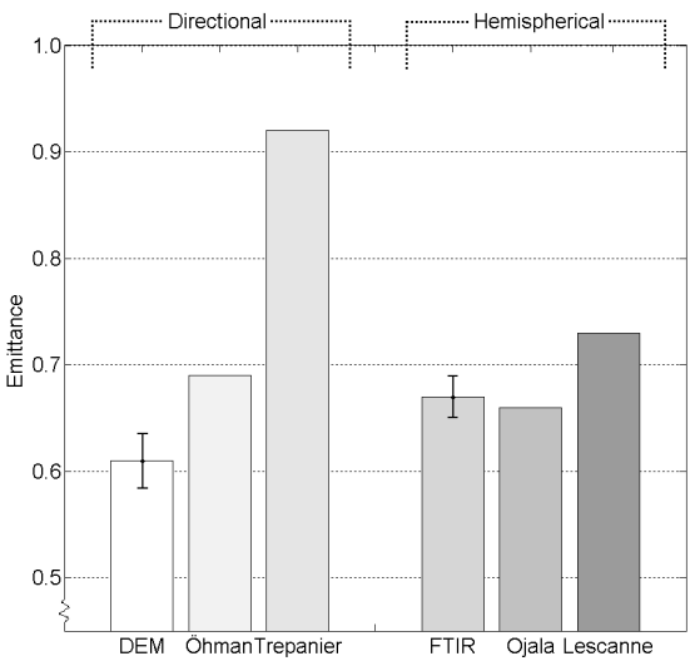

Fig 8. Results from this work and available literature for MWIR wavelengths. Left group of bins: directional emittance. Right group of bins: hemispherical emittance. Values are given for dry paper made of different raw material. Results from this work are given as normal emittance \pm total error. 


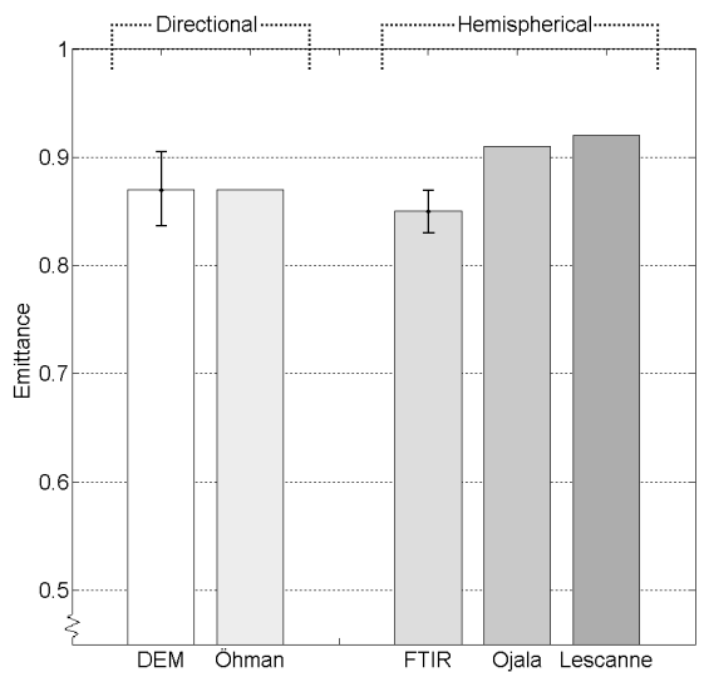

Fig 9. Results from this work and available literature for LWIR wavelengths. Left group of bins: directional emittance. Right group of bins: hemispherical emittance. Values are given for dry paper. Results from this work are given as normal emittance \pm total error.

explanation could be the influence of the ambient radiation from the oven walls in his experimental set-up. In his measurement set-up, the oven walls will have a temperature similar to that of the paper samples (standard newsprint) and thus emit radiation of similar magnitude. If the studied samples has a high reflectance, radiation from the oven walls will be reflected by the sample surface, or absorbed by the sample and then remitted. Both phenomena will lead to a higher amount of radiation detected by the sensor than actually is emitted by the sample itself. Öhman (1999) used a similar methodology as the present study. However, he measured on paperboard and used different emittance values for the Nextel reference.

Results from our study and from the literature for the LWIR wavelength range is shown in Fig 9.

A general conclusion from Fig 9 is that the spread in emittance for different measurement methods and paper types appears to be much smaller if the emittance is determined in the LWIR wavelength range. This implies, that when using a LWIR camera (e.g. microbolometer) in practice, choosing a generic paper emittance should not impart a great error to the quantitative temperature values.

So far there is evidence that the emittance of TMP is significantly higher in the LWIR wavelength range than in the MWIR. It is also demonstrated that the emittance values measured with the DEM method are within $10 \%$ from previously published works for the given wavelength range, with the exception of work by Trepanier (1984). The remaining sections will focus on results from the DEM method where temperature and observation angle has been varied. The influence of the sample temperature on the emittance of TMP is shown in Fig 10.

The temperature dependence of the emittance of the samples is very low. As stated in the introductory section, the emittance of a dielectric is expected to decrease

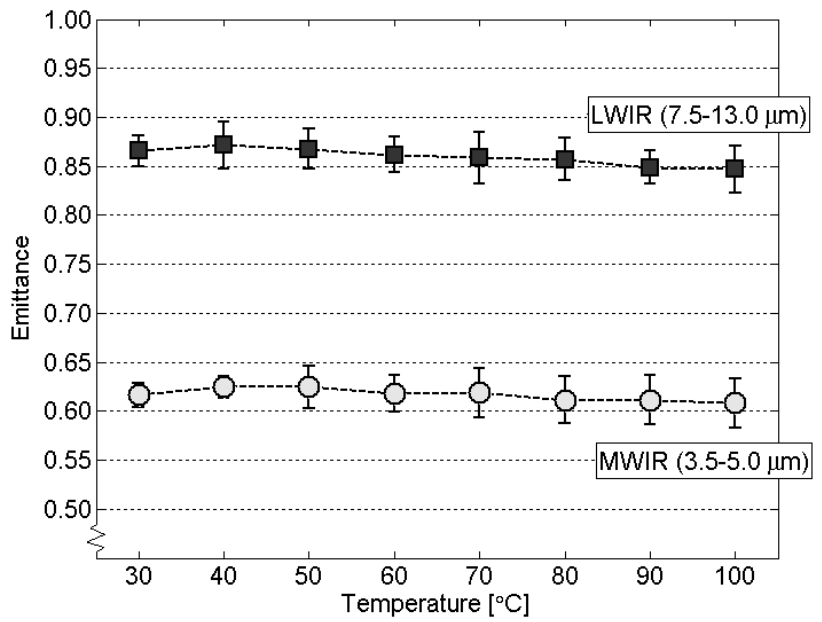

Fig 10. Normal emittance as a function of temperature at two different wavelength ranges. Error bars show the standard deviation of the measurement results.

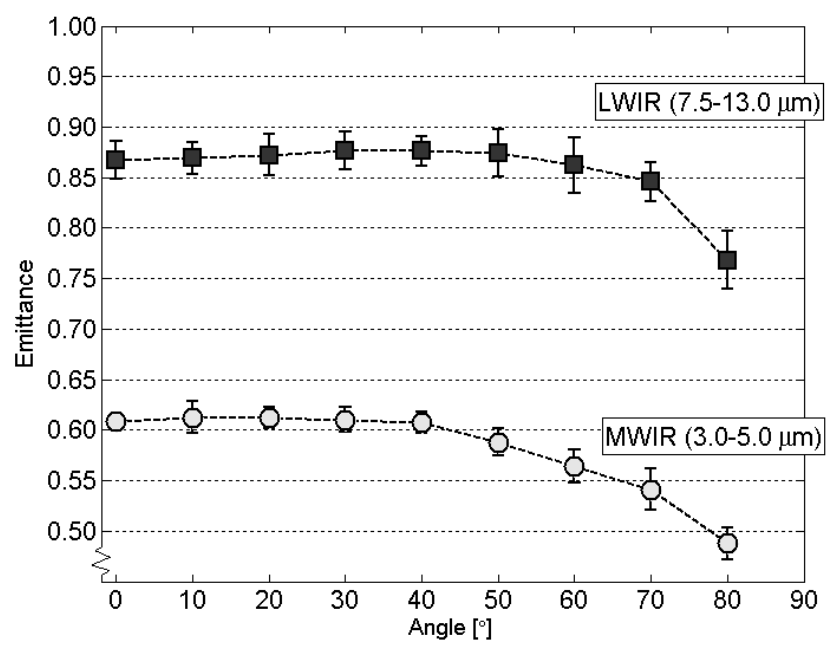

Fig 11. Directional emittance as a function of observation angle for TMP in two different wavelength ranges. An angle of $0^{\circ}$ corresponds to normal observation. Error bars show the standard deviation of the measurement results.

slightly with increased temperature. From the results shown, such a slight decrease could be concluded; however, it is within the error margins.

From the theoretical error propagation one would expect a significant error when the sample temperature is close to the ambient temperature, e.g. for temperatures 30 $50^{\circ} \mathrm{C}$. However this was not seen in the experimental standard deviation. This implies that the experimentally observed error is much better than that predicted by the theoretical error estimation. The results in Fig 10 also confirm that the emittance in the MWIR range is lower than the emittance in the LWIR range.

The influence of the observation angle on the emittance is shown in Fig 11.

The results show that the emittance exhibits an almost constant emittance between angles $0^{\circ}-40^{\circ}$ in the MWIR range and between angles $0^{\circ}-70^{\circ}$ in the LWIR range. The emittance then starts to decrease. Again, this is in good agreement with the expected behaviour of a dielectric. Between $0^{\circ}$ and $80^{\circ}$ the emittance drops by $21 \%$ in the MWIR range and $11 \%$ in the LWIR range. In the LWIR 
range, a slight increase in emittance is noted between angles $30^{\circ}-50^{\circ}$. A similar behaviour was noted by Labed and Stoll (1991) for the emissivity of soils and sands, though the cause remains unexplained.

\section{Summary and conclusions}

A goniometric method for measuring the directional emittance of paper and board samples was developed. Here, the emittance of a sample is determined by comparing its emitted infrared radiance with the radiance emitted from a reference surface with known emittance. The method allows the use of infrared cameras for the measurement of the emittance. In order to investigate the influence of the wavelength range, two cameras, operating in the mid-wavelength (MWIR) and longwavelength infrared (LWIR) range, respectively, were used. Furthermore, the method allows for the adjustment of the observation angle in a range between $0^{\circ}$ (normal to the surface) and approximately $80^{\circ}$. The sample is located in an oven, where it can be heated to a temperature between 30 and $100^{\circ} \mathrm{C}$.

A study was performed to evaluate the method. Here, the directional emittance of handsheets made from thermo-mechanical pulp (TMP) was measured at different wavelengths ranges, sample temperatures and observation angles. The obtained emittance values and trends were in agreement with previous experimental work and theoretical predictions. Furthermore, the emittance of the TMP samples was also measured using an established method based on a hemispherical measurement; FTIR spectroscopy. Given the

\section{Acknowledgements}

The authors of this work would like to thank Prof. Arne Roos at the Solid State Physics group at Uppsala University for his helpful assistance with the FTIR measurements. We would like to thank M.Sc. Claes Öhman for his valuable feedback and for sharing his knowledge, experience and equipment. Furthermore, we would like to thank Magnus Hillergren and Jarmo Tulonen for their help when designing and building the HIP equipment. We gratefully acknowledge the Swedish Energy Agency for the financial support of this PhD project, and Holmen, Korsnäs and Andritz as the financial contributors to the Improved Dewatering Cluster.

\section{Literature}

AGA (1979): AGA Thermovision 780 (en-US) Operating Manual, AGA Infrared Systems, Stockholm, p 110.

AGEMA (1994): ThermoVision System 900 Hardware Manual (en-US), AGEMA Infrared Systems, Stockholm, p 290.

ASTM (2004): Standard Test Method for Determination of Emittance of Materials Near Room Temperature Using Portable Emissometers, ASTM International, United States, p 8.

ASTM (2008): Standard Test Methods for Total Normal Emittance of Surfaces Using Inspection-Meter Techniques, ASTM International, United States, p 3.

Banerjee, D. (2008): Development of High Resolution Optical Measurement Techniques to Investigate Moisture Content and Thermal Properties of Paper, PhD Thesis, Technische Universität Darmstadt, Darmstadt, p 164. methodological differences between the two measurement approaches, the results were in good agreement.

Using the developed method, it is possible to determine accurate quantitative emittance values adapted to a specific camera, observation angle and paper type. The calculated temperature values will therefore be much more accurate, thus allowing for a quantitative analysis of not only the temperatures differences, but also the absolute temperature values for process analysis purposes. In the future, the method will be used to determine the emittance of paper samples made of different furnishes. The influence of moisture content on the emittance will be also investigated. Improvements to the method could be made by successively analyzing the paper sample rows and the emittance reference plate as they are uncovered, instead of analyzing them all in the same image frame. Successive analysis would reduce the error due to cooling. Additionally, using Nextel-painted paper as a reference instead of Nextel-painted aluminium would reduce the error due to the different cooling properties of the paper samples and the reference plate.

In addition to emittance measurements, the developed equipment could also be used for measurement of the directional radiation-related properties in other wavelength ranges. By just exchanging the IR-camera with another camera, for example a camera sensitive in the visual or NIR wavelength range, optical properties can be analyzed as a function of observation angle or temperature in other wavelength ranges.

Banerjee, D., Garbe, C. S., B, S., Jähne, B. and Schabel, S. (2008): An active thermographic technique for highly resolved heat transport measurements in paper drying, Appita Journal 61 (3), 244-249.

Beecroft, M., Szczesniak, M., Smith, B., Matsumoto, F. and Ferguson, J. (2005): Hand-held spectrometer, US Patent 7.236.243 B2, United States.

Bramson, M. A. (1968): Infrared Radiation - A Handbook for Applications, Honeywell Inc. Radiation Center, Boston, MA, p 623.

Charles, J. A. (1999): Line-scan infrared imaging for papermachine diagnostics, 1999 TAPPI Engineering Conference, Anaheim, California, 10.

Dewitt, D. P. and Nutter, G. D. (1988): Theory and Practice of Radiation Thermometry, Wiley-Interscience, New York, p 1138.

Dumbleton, D., Kringstad, K. and Söremark, C. (1973): Temperature profiles in paper during straining, Svensk Papperstid. (14), 521-528.

Fike, G. (2004): Using Infrared Thermography to image the drying of polymer surfaces, Master Thesis, Georgia Institute of Technology, p 67.

Gaussorgues, G. (1994): Infrared Thermography, Chapman \& Hall, Cambridge, UK, p 529.

Griffiths, P. and De Haseth, J. A. (1986): Fourier Transform Infrared Spectrometry, Wiley-Interscience, p 672.

Hojjatie, B., Abedi, J. and Coffin, D. W. (2001): Quantitative determination of in-plane moisture distribution in paper by infrared thermography, Tappi J. 84 (5), 11. 
Hyll, C., Vomhoff, H. and Mattsson, L. (2011): A method for measurement of the directional emissivity of paper; Innventia Report 286, Innventia AB, Stockholm, p 40.

Kiiskinen, H. and Pakarinen, P. (1998): Infrared thermography at examination of paper structure, Proceedings of SPIE 3361, 6. Koirala, L. R. (2004): FTIR-Spectroscopic Measurement of Directional Spectral Emissivities of Microstructured Surfaces, PhD Thesis, Helmut-Schmidt University, Hamburg, p 245.

Kwor, E. T. and Matteï, S. (2001): Emissivity measurements for Nextel Velvet Coating 811-21 between -36 degC and 82 $\operatorname{deg}$, High temperatures - high pressures 33, 551-557.

Labed, J. and Stoll, P. (1991): Angular variation of land surface spectral emissivity in the thermal infrared: laboratory investigations on bare soils, International Journal of Remote Sensing 12 (11), 2299-2310.

Lescanne, Y. (1992): Caracterisation du papier en vue de la simulation du sechage par infrarouge electrique, L'Institut National Polytechnique de Lorraine, Vandoeuvre-lès-Nancy, $p$ 202.

Madding, R. P. (1999): Emissivity measurement and temperature correction accuracy and considerations, SPIE (ed.), SPIE Conference on Thermosense XXI, Orlando, FL, 393-400.

Maldague, X. P. V. (2001): Theory and practice of infrared technology for nondestructive testing, Wiley, p 684.

McCluney, W. R. (1994): Introduction to Radiometry and Photometry, Artech House, Boston, p 424.

Minkina, W. and Dudzik, S. (2009): Infrared Thermography Errors and Uncertainties, John Wiley \& Sons Ltd, Chichester, West Sussex, UK, p 190.

Ojala, K. (1993): Studies on infrared drying of paper, use of integrating spheres in FTIR-measurements, and heat and mass transfer inside paper, $\mathrm{PhD}$ Thesis, University of Helsinki, Espoo, p 70.

Ojala, K. T., Koski, E. and Lampinen, M. (1992): Reflection and transmission measurements with an integrating sphere and Fourier-transform infrared spectrometer, Applied Optics 31 (22), 4582-4589.

Puram, C., Daryabeigi, K., Wright, R. and Alderfer, D. (1994): Directional Emittance Surface Measurement System and Process, US Patent 5.347.128, United States.

Sheahen, T. P. (1978): Use of infra-red thermography in pulp and paper mills, TAPPI ANNUAL MEETING (Chicago), 1.

Smith, B. C. (2011): Fundamentals of Fourier Transform Infrared Spectroscopy, CRC Press, p 207.

Trepanier, R. J. (1984): The infrared emissivity of wet webs, Journal of Pulp and Paper Science (November 1984), J166J172.

Vickery, D. E., Luce, J. E. and Atkins, J. W. (1978): Infrared thermography - An Aid to solving paper machine moisture profile problems, Tappi Papermakers Conference, 39-51.

Vollmer, M. and Möllmann, K.-P. (2010): Infrared Thermal Imaging: Fundamentals, Research and Applications, WileyVCH, Weinheim, Germany, p 593.

Yamauchi, T. and Murakami, K. (1992): Observation of deforming and fracturing processes of paper by using infrared thermography, Japan Tappi 4, 70-75.

Öhman, C. (1996): Equalizing Box User's Reference Manual, AGEMA Infrared Systems, Danderyd, p 10.

Öhman, C. (1999): Emittansmätningar med AGEMA E-Box, Teknisk rapport, Stockholm, p 19.

Öhman, C. (2001): Measurement in thermography, Infrared Training Center, Stockholm, p 114.

Manuscript received April 10, 2012 Accepted August 24, 2012 